\title{
Average lattice symmetry and nanoscale structural correlations in magnetoresistive manganites
}

\author{
V. Kiryukhin ${ }^{1}$, T. Y. Koo ${ }^{1}$, H. Ishibashi ${ }^{1,2}$, J. P. Hill ${ }^{3}$, and S-W. Cheong ${ }^{1}$ \\ (1) Department of Physics and Astronomy, Rutgers University, Piscataway, New Jersey 08854 \\ (2) Department of Materials Science, Osaka Prefecture University, Sakai, Osaka 599-8531, Japan \\ (3) Department of Physics, Brookhaven National Laboratory, Upton, New York 11973
}

(October 29, 2018)

\begin{abstract}
We report $\mathrm{x}$-ray scattering studies of nanoscale structural correlations in the paramagnetic phases of the perovskite manganites $\mathrm{La}_{0.75}\left(\mathrm{Ca}_{0.45} \mathrm{Sr}_{0.55}\right)_{0.25} \mathrm{MnO}_{3}, \mathrm{La}_{0.625} \mathrm{Sr}_{0.375} \mathrm{MnO}_{3}$, and $\mathrm{Nd}_{0.45} \mathrm{Sr}_{0.55} \mathrm{MnO}_{3}$. We find that these correlations are present in the orthorhombic $O$ phase in $\mathrm{La}_{0.75}\left(\mathrm{Ca}_{0.45} \mathrm{Sr}_{0.55}\right)_{0.25} \mathrm{MnO}_{3}$, but they disappear abruptly at the orthorhombic-to-rhombohedral transition in this compound. The orthorhombic phase exhibits increased electrical resistivity and reduced ferromagnetic coupling, in agreement with the association of the nanoscale correlations with insulating regions. In contrast, the correlations were not detected in the two other compounds, which exhibit rhombohedral and tetragonal phases. Based on these results, as well as on previously published work, we propose that the local structure of the paramagnetic phase correlates strongly with the average lattice symmetry, and that the nanoscale correlations are an important factor distinguishing the insulating and the metallic phases in these compounds.
\end{abstract}

PACS numbers: 75.30.Vn, 71.30.+h, 71.38.-k

The physical mechanism underlying the magneticfield-induced insulator-metal transition in perovskite manganites $A_{1-x} B_{x} \mathrm{MnO}_{3}$ has been the subject of intense experimental and theoretical investigation since its rediscovery in $1993[1,2]$. One of the motivations for such considerable attention is the unusually large diminution of the electrical resistivity observed at the magnetic-fieldinduced transition, which is now commonly referred to as the colossal magnetoresistance (CMR). Several different kinds of the CMR effect are known [2]. The most widely studied variant of this effect is the transition from a paramagnetic insulating (PI) to a ferromagnetic metallic (FM) phase. The large difference between the resistivities of these two phases lies at the heart of the CMR effect. The metallic nature of the FM phase has been explained within the framework of the double-exchange mechanism [2]. However, the physical mechanism responsible for the large resistivity of the PI phase remains poorly understood.

The situation is complicated significantly by the fact that transport properties of the paramagnetic state in manganites with the same doping level $x$, but with different cations $A$ and $B$, are often very different. For example, the electrical resistivity of $\mathrm{La}_{0.7} \mathrm{Sr}_{0.3} \mathrm{MnO}_{3}$ shows a metallic behavior (i.e. grows with temperature) [3], while the resistivity of $\mathrm{La}_{0.7} \mathrm{Ca}_{0.3} \mathrm{MnO}_{3}$ exhibits the temperature dependence typical of an insulator, decreasing with temperature [4]. As a consequence, the resistivity of the latter compound is significantly larger than that of the former. These differences cannot be explained by steric modification of the electronic bandwidth $W$ due to the evolution of the average structural parameters from one composition to another as a result of the differing size of the divalent dopants $[5,6]$. In fact, the value of $W$ in $\mathrm{La}_{0.7} \mathrm{Ca}_{0.3} \mathrm{MnO}_{3}$ is expected to differ from that in $\mathrm{La}_{0.7} \mathrm{Sr}_{0.3} \mathrm{MnO}_{3}$ by less than $1 \%$ as a result of such effects [5]. The widely accepted solution to this problem, first proposed theoretically [7], is that the electron-phonon coupling $\lambda$ plays an essential role. The resistivity is expected to be larger in compounds with large values of $\lambda$.

There is a significant amount of experimental evidence that electron-phonon coupling plays a key role in the manganites. It is well established that small lattice polarons are present in the PI state [8-11]. A lattice polaron forms when an $e_{g}$ electron localizes on a $\mathrm{Mn}^{3+}$ ion, and the surrounding oxygen octahedron distorts due to the Jahn-Teller effect. Formation of lattice polarons leads to an increase of the electrical resistivity. In addition to single polarons, the paramagnetic state exhibits correlated lattice distortions with a correlation length of several lattice constants [12]. It has been proposed that the structural correlations reported in Ref. [12] reflect the presence of nanoscale regions possessing charge and orbital order, and that these regions are responsible for the enhanced resistivity of the PI phase. The temperature dependence of the electrical resistivity has been shown to follow the concentration of the correlated regions [12], in agreement with this hypothesis. Further, the correlations are strongly suppressed in an applied magnetic field [13], implying that they play an important role in the CMR effect. The detailed structure of the correlated regions remains to be elucidated [14], but recent experimental studies suggest $[15,16]$ that the correlated regions exhibit CE-type and striped charge and orbital ordered structures similar to those found in manganites possess- 
ing long-range charge and orbital order [17]. Numerous theoretical works have also been devoted to the problem of structural and electronic inhomogeneities in manganites [18], but no realistic description of the PI state has been achieved thus far.

In the above scenario, the differences in the transport properties of the PI state in various manganites must then be explained by variations in the concentration, size, or other properties of the correlated regions. Such variations might, in principle, result from a dependence of the electron-phonon coupling $\lambda$ on sample composition. However, $\lambda$ is not expected to depend significantly on the $A$-site ionic composition (because it is, essentially, a local parameter proportional to the Jahn-Teller energy $E_{J T}$ ). Thus, other reasons for the observed variations of physical properties must be identified. Among the possible explanations, it has been proposed, for example, that in manganites with a large variance in the $A$ cation radius, cation size disorder could produce strain fields stabilizing local Jahn-Teller distortions [19]. This explanation does not work, however, in the case of the $\mathrm{La}_{0.7} \mathrm{Sr}_{0.3} \mathrm{MnO}_{3}$ and $\mathrm{La}_{0.7} \mathrm{Ca}_{0.3} \mathrm{MnO}_{3}$ compounds discussed above, since the metallic Sr compound exhibits larger $A$-site cation radius variation than the $\mathrm{Ca}$ compound, which is insulating at high temperatures.

In this paper, we discuss a different scenario. Specifically, we point out that the symmetry of the average structure can affect the energetics for the formation of local distortions by allowing certain types of distortions to occur more easily, or, alternatively, by suppressing them. The importance of the average long-range structure stems from the fact that the significant lattice distortions associated with the correlated regions give rise to long-range anisotropic strain [20]. Thus, lattice symmetry is one of the important factors determining how easily the local distortions are accommodated in a given crystal structure. Manganites exhibit structural transitions between distorted perovskite phases possessing orthorhombic, rhombohedral, tetragonal, and monoclinic lattice symmetry. As explained above, it is reasonable to expect that some of these phases will be more susceptible towards formation of local distortions than others.

In this work, therefore, we investigate the connection between the average lattice symmetry and the local (nanoscale) correlations in several perovskite manganite compounds. X-ray scattering studies of the structural correlations in manganites exhibiting orthorhombic, rhombohedral, and tetragonal structural phases are reported. We find that in the samples investigated, as well as in the previously studied manganites, these correlations are present only in the orthorhombic $O$ phase. This result is most convincingly demonstrated in experiments with $\mathrm{La}_{0.75}\left(\mathrm{Ca}_{0.45} \mathrm{Sr}_{0.55}\right)_{0.25} \mathrm{MnO}_{3}$. This compound undergoes an orthorhombic-to-rhombohedral transition with increasing temperature. We find that the structural correlations abruptly disappear at the tran- sition to the rhombohedral state. Based on these results and on previously published work, we propose that the correlations form most easily in the orthorhombic $O$ phase. In this phase, the crystal lattice is contracted along the $c$ axis, as is also the case in structures which exhibit charge-ordered phases, in which the long axes of the $\mathrm{Mn}^{3+} \mathrm{O}_{6}$ octahedra lie in the $a b$ plane. In contrast, the correlations are suppressed in rhombohedral and tetragonal phases, in which the $\mathrm{MnO}_{6}$ octahedra are either undistorted, or elongated along the $c$ axis, on average. Thus, the local structure of the paramagnetic phase and, consequently, its physical properties do, in fact, correlate strongly with the average lattice symmetry.

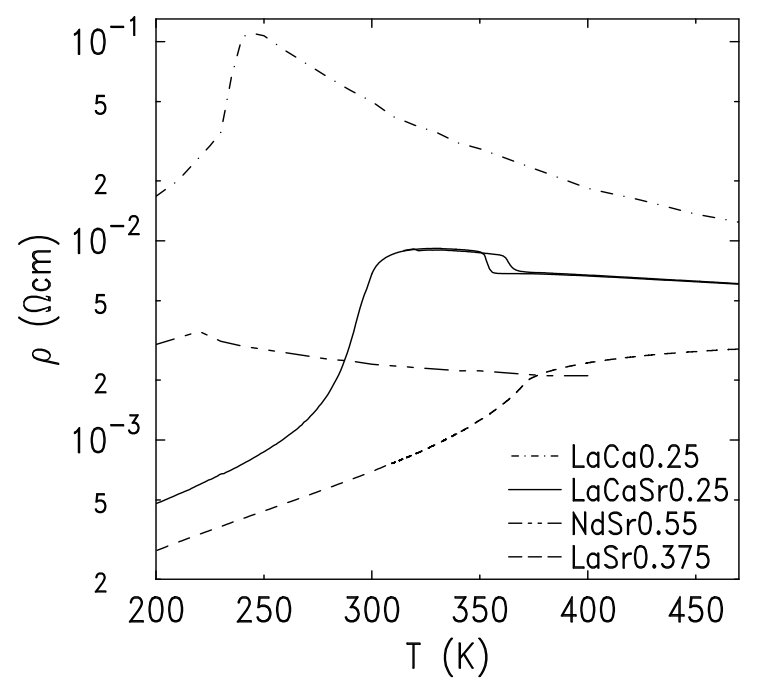

FIG. 1. Temperature dependence of the electrical resistivity of $\mathrm{La}_{0.75} \mathrm{Ca}_{0 .}{ }_{25} \mathrm{MnO}_{3}$, $\mathrm{La}_{0.75}\left(\mathrm{Ca}_{0.45} \mathrm{Sr}_{0.55}\right)_{0.25} \mathrm{MnO}_{3}, \mathrm{Nd}_{0.45} \mathrm{Sr}_{0.55} \mathrm{MnO}_{3}$ (taken from Ref. [21]), and $\mathrm{La}_{0.625} \mathrm{Sr}_{0.375} \mathrm{MnO}_{3}$.

Single crystals of $\mathrm{La}_{0.75}\left(\mathrm{Ca}_{0.45} \mathrm{Sr}_{0.55}\right)_{0.25} \mathrm{MnO}_{3}$ (LCSMO), $\mathrm{La}_{0.625} \mathrm{Sr}_{0.375} \mathrm{MnO}_{3}$, and $\mathrm{Nd}_{0.45} \mathrm{Sr}_{0.55} \mathrm{MnO}_{3}$ were grown using the floating zone technique. X-ray diffraction measurements were carried out at beamline X22C at the National Synchrotron Light Source. A 10.3-keV x-ray beam was focused by a mirror, monochromatized by a double-crystal Ge (111) monochromator, scattered from the sample, and analyzed with a pyrolytic graphite crystal. The samples were mounted in a closed-cycle refrigerator $(\mathrm{T}=6-450 \mathrm{~K})$. In this paper, Bragg peaks are indexed in the orthorhombic Pbnm notation, in which the $a, b$, and $c$ axes run along the $(1,1,0),(1,-1,0)$, and $(0,0,1)$ cubic perovskite directions, respectively. Scattering vectors $(h, k, l)$ are given in reciprocal lattice units.

We first focus our discussion on the properties of the LCSMO sample. Its high temperature PI state exhibits rhombohedral symmetry. With decreasing temperature, this compound first undergoes a first order structural transition into the orthorhombic PI phase at $T_{s} \approx 360$ $\mathrm{K}$, and then becomes a ferromagnetic metal at $T_{c} \approx 300$ 
K. These transitions are clearly reflected in the behavior of the electrical resistivity, shown as solid line in Fig. 1. The x-ray scattering patterns around the $(4,4,0)$ Bragg peak in the orthorhombic $(T=300 \mathrm{~K})$ and rhombohedral $(T=400 \mathrm{~K})$ phases are shown in Fig. 2. The sharp peaks at $(4.5,3.5,0)$ and $(3.5,4.5,0)$ at $T=300 \mathrm{~K}$ arise from crystallographic twinning in the orthorhombic phase. The "butterfly"-shaped feature in the center is commonly attributed to scattering from uncorrelated polarons, also known as Huang scattering, together with thermal-diffuse scattering [10]. At $T=300 \mathrm{~K}$, there are also 4 broad peaks located at $(4 \pm 0.5,4,0)$ and $(4,4 \pm 0.5,0)$. These peaks are best seen in scans along the $h$ or $k$ directions. One such scan at $T=320 \mathrm{~K}$ is shown in Fig. $3(\mathrm{a})$.

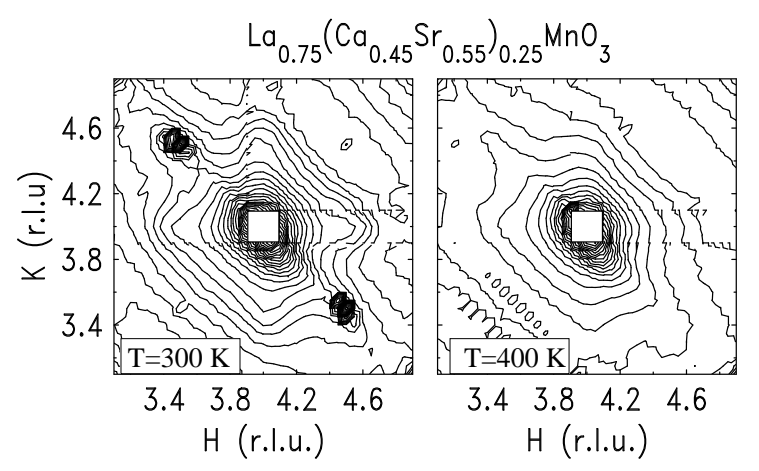

FIG. 2. Contour plots of the x-ray intensity around the $(4,4,0)$

Bragg peak at $T=300 \mathrm{~K}$ (orthorhombic phase) and $T=400$ $\mathrm{K}$ (rhombohedral phase) in $\mathrm{La}_{0.75}\left(\mathrm{Ca}_{0.45} \mathrm{Sr}_{0.55}\right)_{0.25} \mathrm{MnO}_{3}$.

The broad peaks at $(4 \pm 0.5,4,0)$ and $(4,4 \pm 0.5,0)$ reflect the presence of nanoscale correlated regions which are believed to possess charge and orbital order [12]. The intensity of these peaks reflects the concentration of the correlated regions [15]. To study the temperature dependence of this concentration, we took data at different temperatures. Some of these data are shown in Fig. $3(\mathrm{a})$. The data were fitted to a sum of a Gaussian line shape and a monotonically sloping background, the latter described by a power-law function. This background contains contributions from single polaron and thermaldiffuse scattering [10]. The error bars were estimated as described in Ref. [15].

The intensity of the correlated peak in LCSMO as a function of temperature is shown in Fig. 4(a). The central observation of this work is that the correlated regions abruptly disappear at the transition from the orthorhombic to rhombohedral phase. Thus, in LCSMO, the structural correlations are present only in the orthorhombic state. The data of Fig. 4(a) show that the correlated regions also disappear in the FM state, as was reported in earlier works $[12,15]$. However, in contrast to the FM state, the rhombohedral phase is insulating. Therefore, the suppression of the correlated insulating regions in this phase is a much more surprising result.

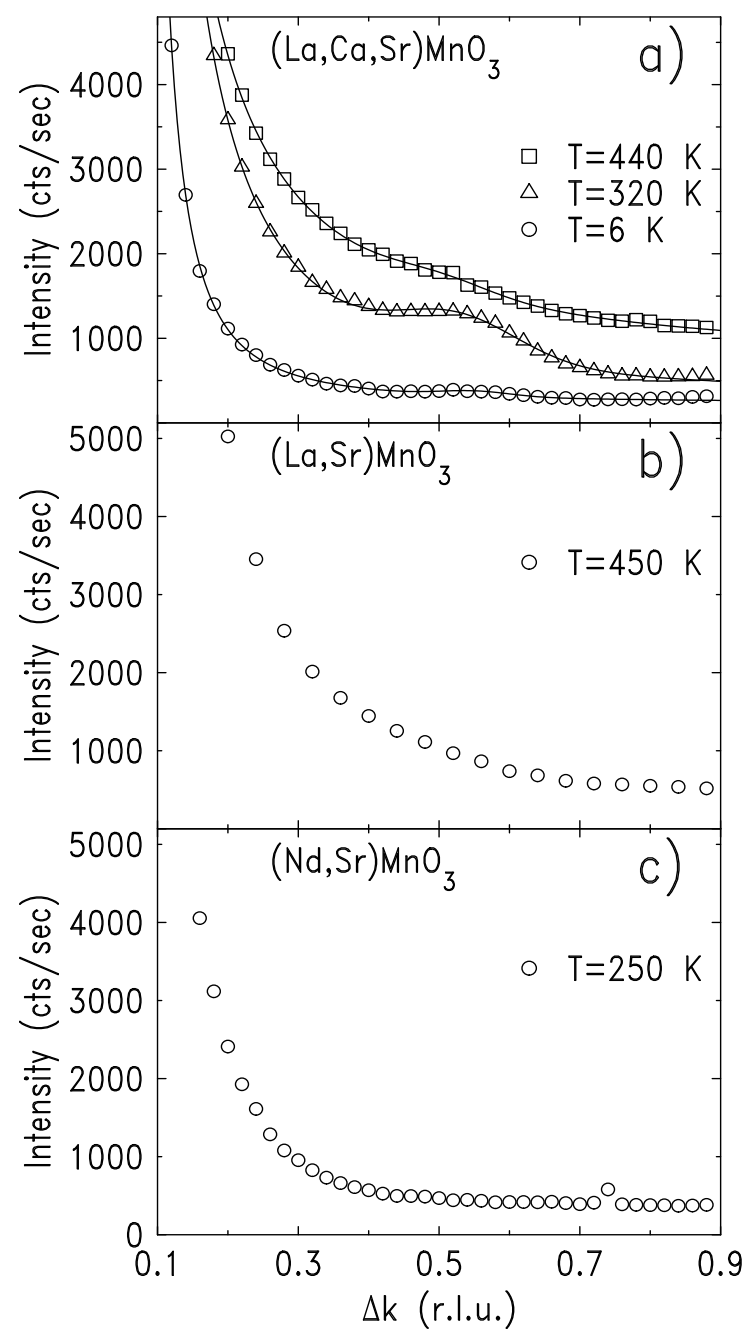

FIG. 3. X-ray scans along the $(4,4+\Delta k, 0)$ direction in $\mathrm{La}_{0.75}\left(\mathrm{Ca}_{0.45} \mathrm{Sr}_{0.55}\right)_{0.25} \mathrm{MnO}_{3}$ (a), along $(6,2+\Delta k, 0)$ in $\mathrm{La}_{0.625} \mathrm{Sr}_{0.375} \mathrm{MnO}_{3}$ (b), and along $(6,4+\Delta k, 0)$ in $\mathrm{Nd}_{0.45} \mathrm{Sr}_{0.55} \mathrm{MnO}_{3}$ (c). The solid lines are the results of fits, as described in the text.

Fig. 4(b) shows that the electrical resistivity abruptly decreases and the magnetic susceptibility increases as the sample enters the rhombohedral phase and the correlated regions disappear. The Curie temperature and the effective magnetic moment extracted from the susceptibility data are $T_{c}=302(2) \mathrm{K}, p=6.2$, and $T_{c}=322(3) \mathrm{K}, p=5.6$ for the orthorhombic and the rhombohedral states, respectively. Thus, the orthorhombic state possesses the larger effective moment, but smaller ferromagnetic exchange constant than the corresponding values in the rhombohedral state. This observation is in agreement with the proposed CE-type charge and orbital ordered structure of the correlated regions, which should be insulating and exhibit antiferromagnetic correlations, pro- 
vided that these regions are sufficiently large $[15,16]$. We note, however, that neutron scattering experiments have thus far failed to detect the CE-type antiferromagnetic correlations in the PI state [12], and therefore the character of the magnetic interaction in the correlated regions is yet to be established experimentally. Alternatively, the increased effective ferromagnetic exchange constant of the more conducting rhombohedral phase can stem from the effects of double exchange which favors ferromagnetic correlations.

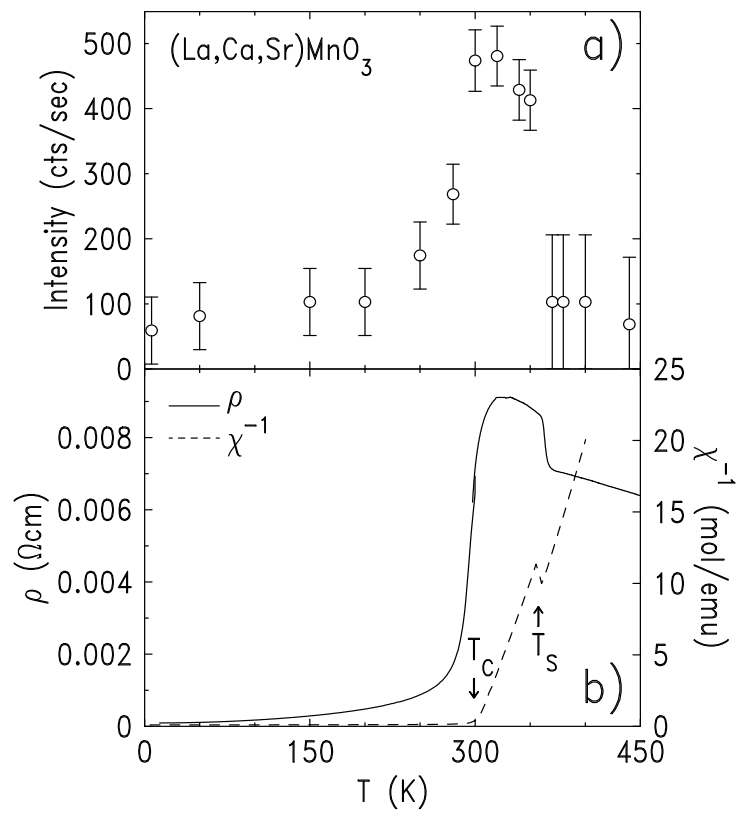

FIG. 4. (a) Temperature dependence of the intensity of the peak due to the structural correlations in $\mathrm{La}_{0.75}\left(\mathrm{Ca}_{0.45} \mathrm{Sr}_{0.55}\right)_{0.25} \mathrm{MnO}_{3}$. The single polaron background is subtracted as discussed in the text. (b) Temperature dependence of the electrical resistivity (solid line), and inverse magnetic susceptibility (dashed line). The data were taken on heating.

The ratio of the integrated intensity of the $(4,4.5,0)$ peak to the integrated intensity of the main Bragg $(4,4,0)$ peak in LCSMO is of the order of $10^{-5}$. In the long-range charge-ordered phases, such as that in $\mathrm{La}_{0.5} \mathrm{Ca}_{0.5} \mathrm{MnO}_{3}$, the typical intensity ratio between the brightest chargeordering peaks and Bragg peaks is of the order of $10^{-2}$ (Ref. [22]). This is significantly larger than what is observed in LCSMO. One can estimate the intensity ratio of the $(4,4.5,0)$ and the $(4,4,0)$ peaks using the structural models proposed in Ref. [22] (the CE-type charge and orbital ordered structure) for $\mathrm{La}_{0.5} \mathrm{Ca}_{0.5} \mathrm{MnO}_{3}$, and in Ref. [17] (the ordering of Zener polarons) for $\operatorname{Pr}_{0.6} \mathrm{Ca}_{0.4} \mathrm{MnO}_{3}$. The calculated ratios are $2.7 \times 10^{-2}$ for the former, and $4.1 \times 10^{-2}$ for the latter model structure. Assuming that the correlated regions in LCSMO are small charge ordered regions with the structure identical to the chargeordered state in $\mathrm{La}_{0.5} \mathrm{Ca}_{0.5} \mathrm{MnO}_{3}$, we obtain the result that the volume fraction of these regions (defined here as the fraction of $\mathrm{MnO}_{6}$ octahedra experiencing the correlated distortion) is smaller than $0.1 \%$. In contrast, a much larger estimate of "a few percent" was obtained for the same fraction in a recent electron diffraction study of $\mathrm{La}_{2 / 3} \mathrm{Ca}_{1 / 3} \mathrm{MnO}_{3}$ [16]. The reason for such a discrepancy is currently unclear. In either case, it is hard to see how such an apparently small volume fraction of the charge and orbital ordered insulating phase could significantly affect the transport properties.

Thus, these observations appear to contradict the strong correlation between the nanoscale correlations and the transport properties observed in a large number of manganites of different chemical compositions and doping levels $[12,13,15,16]$. One way to explain this contradiction is to assume that the lattice distortions are significantly (at least a factor of 10) smaller in the correlated regions than they are in the corresponding longrange ordered phases. Since the diffraction signal from the correlated regions is proportional to the square of the magnitude of the lattice distortion, much larger volume fractions of the charge and orbital ordered insulating phase are then deduced from the experimental data under this assumption.

Alternatively, more elaborate models of the inhomogeneous PI state should be proposed. In particular, dynamic inhomogeneities might play an important role in this state, as evidenced by recent experiments in the related bi-layered manganites [23], in which the structural correlations were found to become completely dynamic at high temperatures. Also, the role of the uncorrelated local distortions must be clarified. The latter distortions are known to be present in both the rhombohedral and the orthorhombic manganites, and to have composition-independent distortion magnitude [11]. In the orthorhombic $\mathrm{La}_{1-x} \mathrm{Ca}_{x} \mathrm{MnO}_{3}$ system, the number of $\mathrm{MnO}_{6}$ octahedra experiencing a distortion (correlated or otherwise) was found to be functionally correlated to the magnetic and transport properties $[9,24]$. The relative contributions of the correlated and uncorrelated distortions to these functional relationships remain to be characterized. It also remains to be seen whether these relationships hold on the rhombohedral side of the structural transition boundary. In this work, we do not attempt to characterize the uncorrelated distortions because it is difficult to distinguish scattering due to these distortions from thermal-diffuse scattering in our energy-integrating measurements. Thus, while it is clear that the correlations play an important role in the manganites, more work is needed to elucidate the microscopic structure of the PI state.

We next briefly discuss the other samples. $\mathrm{La}_{0.625} \mathrm{Sr}_{0.375} \mathrm{MnO}_{3}$ is rhombohedral (space group $R \overline{3} c$ ) at all temperatures and exhibits a paramagnetic state for $T>370 \mathrm{~K}$. The paramagnetic insulating state of $\mathrm{Nd}_{0.45} \mathrm{Sr}_{0.55} \mathrm{MnO}_{3}$ is tetragonal (space group $I 4 / \mathrm{mcm}$ ) 
[26]. In the latter sample, the PI state is observed for $T>T_{N} \approx 220 \mathrm{~K}$. The electrical resistivities of these samples are shown in Fig. 1. In the both samples, we did not detect any evidence of the structural correlations at any temperature. Figs. 3(b,c) show some of the collected data for these samples (Bragg peaks are again indexed using the orthorhombic notation). In contrast, these correlations are clearly present in the paramagnetic orthorhombic phase of $\mathrm{La}_{0.75}\left(\mathrm{Ca}_{0.45} \mathrm{Sr}_{0.55}\right)_{0.25} \mathrm{MnO}_{3}$ discussed above, and also in the PI phases of numerous other orthorhombic manganites, such as $\mathrm{Nd}_{1-x} \mathrm{Sr}_{x} \mathrm{MnO}_{3}$ $(0.3 \leq x \leq 0.5)$ [15], $\mathrm{La}_{1-x} \mathrm{Ca}_{x} \mathrm{MnO}_{3}(0.2 \leq x \leq 0.3)$ [12], and $\mathrm{Pr}_{1-x} \mathrm{Ca}_{x} \mathrm{MnO}_{3}(0.3 \leq x \leq 0.5)$ [25]. We suggest then that the nanoscale structural correlations described above can only occur in an orthorhombic phase. Of course, more systematic studies are needed to verify this intriguing hypothesis, but even absent such studies, we feel that there is sufficient evidence in hand to warrant speculation as to why such an observation might be true.

All the orthorhombic samples discussed above exhibit the well known $O$ structure with the lattice constants $c / \sqrt{2}<b \sim a$ [26,27]. An orthorhombic state with $c / \sqrt{2}<b \sim a$ is also realized in the manganites with long-range CE-type charge and orbital order. In this structure, the $c$-axis contraction reflects the global effect of ordered Jahn-Teller distortions of the $\mathrm{Mn}^{3+} \mathrm{O}_{6}$ octahedra with the long axis lying in the $a b$ plane [2]. Thus, on average, the long axes of the $\mathrm{MnO}_{6}$ octahedra lie in the $a b$ plane in both the long-range charge ordered phase, and in the $O$ paramagnetic insulating state $[26,27]$. In contrast, the $\mathrm{MnO}_{6}$ octahedra are undistorted in the rhombohedral $R \overline{3} c$ state [28], and are significantly elongated along the $c$-axis in the tetragonal $I 4 / \mathrm{mcm}$ state [26].

It is not completely surprising that the $\mathrm{MnO}_{6}$ octahedra are distorted in a similar manner in the manganites exhibiting nanoscale charge-ordered regions and in the manganites with long-range charge and orbital order. Note, however, that this is a quite nontrivial result because there is no a priori requirement that the local symmetry of the nanoscale ordered regions should match the average symmetry of the crystal lattice. The energetically favorable response of the orthorhombic structure to the long-range strains produced by the local distortions is likely to be one of the key factors explaining the reported observations. Theoretical calculations taking into account the details of the lattice structure are needed to confirm these ideas. In any case, the proposed correlation between the presence of the local lattice distortions and the average crystallographic structure is intriguing and deserves further theoretical and experimental investigation.

Finally, we return to the problem of the drastic variation of the electrical properties of the paramagnetic phase with chemical composition. We emphasize that here we are discussing the high-temperature paramagnetic phase, and not the low-temperature state. Specifically, we will consider the $\mathrm{La}_{1-x}\left(\mathrm{Ca}_{1-y} \mathrm{Sr}_{y}\right)_{x} \mathrm{MnO}_{3}$ series of compounds with $x$ in the vicinity of 0.3 . This system exhibits a well defined insulating behavior for $y=0$ (see Fig. 1), and is metallic for $y=1$ (Fig. 1, and Ref. [3]). At fixed $x$ and with $y$ increasing from 0 to 1 , the system should, therefore, undergo an insulator-to-metal transition. It is well known that with $y$ increasing from 0 to 1 , this system also undergoes an orthorhombic-to-rhombohedral structural transition [29]. With the exception of the narrow region in the vicinity of the structural transition, the electrical resistivity shows the temperature dependence typical for an insulator in the orthorhombic samples, and that of a metal in the rhombohedral state [29]. These data show that the metal-insulator transition coincides approximately with the structural transition in this series of compounds.

In the vicinity of the structural transition, the electrical resistivity exhibits temperature-dependent behavior typical of an insulator in the both structural phases, see Fig. 4(b), and Ref. [29]. We would like to point out an interesting possibility that the insulating behavior of the rhombohedral phase could result from the effects of chemical and magnetic disorder. These effects are, in fact, well known to play an important role in mixed-valence manganites [2]. In the $\mathrm{La}_{1-x}\left(\mathrm{Ca}_{1-y} \mathrm{Sr}_{y}\right)_{x} \mathrm{MnO}_{3}$ series of compounds, in particular, the importance of disorder is evidenced by the large magnitude of the electrical resistivity deep in the paramagnetic metallic region of the phase diagram [29]. It is, therefore, reasonable to expect that in samples with less chemical disorder, the metal-insulator transition boundary lies closer or even coincides with the structural transition boundary. We, therefore, believe that further theoretical and experimental investigation of the role of disorder in the $\mathrm{La}_{1-x}\left(\mathrm{Ca}_{1-y} \mathrm{Sr}_{y}\right)_{x} \mathrm{MnO}_{3}$ series of compounds is of great interest.

The experimental data for one of the compounds from this series, $\mathrm{La}_{0.75}\left(\mathrm{Ca}_{0.45} \mathrm{Sr}_{0.55}\right)_{0.25} \mathrm{MnO}_{3}$, were discussed in detail above. Our experiments clearly show that the nanoscale structural correlations are present only on the orthorhombic side of the phase boundary. In addition, these correlations are clearly present in the $y=0 \mathrm{com}$ pound $\mathrm{La}_{0.75} \mathrm{Ca}_{0.25} \mathrm{MnO}_{3}$ [12], and are undetectable in the $y=1$ material $\mathrm{La}_{0.625} \mathrm{Sr}_{0.375} \mathrm{MnO}_{3}$. Thus, the existing experimental data show that the metallic rhombohedral state in this compound series does not exhibit the nanoscale correlations, while the correlations are present in the insulating orthorhombic state. The presence of the nanoscale insulating regions, therefore, appears to be an important factor distinguishing the insulating and the metallic phases. Thus, the nanoscale correlations should be included in any physical mechanism explaining the observed large variation of the high-temperature properties of manganites and, consequently, the observed values of the CMR effect.

In summary, we report x-ray scattering studies of nanoscale structural correlations in several manganite 
compounds exhibiting orthorhombic, rhombohedral, and tetragonal perovskite phases. Based on these results, and also on previously published work, we propose that these correlations form most easily in the orthorhombic $O$ phase, and are suppressed in the rhombohedral and the tetragonal phases. Thus, the local structure of the paramagnetic phase appears to correlate strongly with the average lattice symmetry. Our experiments strongly suggest that changes in physical properties observed at structural transitions in manganites cannot be understood through spatially uniform phases, and that nanoscale inhomogeneities must play an essential role in these transitions.

Note added: A recent work by Mira at al. reports measurements of thermal properties, magnetoresistance, and lattice expansion in various manganites [30]. The analysis of these quantities led the authors of Ref. [30] to a conclusion that lattice effects (electron-lattice interaction, and local Jahn-Teller distortions) are important in the orthorhombic manganites, while these effects are strongly reduced in the rhombohedral samples. The orthorhombic PI state was proposed to contain a superparamagnetic secondary phase in a certain temperature interval above $T_{c}$. These results are consistent with the $\mathrm{x}$-ray diffraction measurements reported in our paper, providing further evidence of the correlation between the local structure and the average lattice symmetry, and thus revealing the physics underlying the drastic differences in the properties of manganites with different lattice symmetry.

We are grateful to K. I. Kugel, A. J. Millis, and J. Mira for important discussions. This work was supported by the NSF under grants No. DMR-0093143, and DMR0103858, by the DOE under Contract No. DE-AC0298CH10886, and by the NSF MRSEC program, Grant No. DMR-0080008.

[1] S. Chahara, T. Ohno, K. Kasai, Y. Kozono, Appl. Phys. Lett. 63, 1990 (1993); R. von Helmolt, J. Wecker, B. Holzapfel, L. Schultz, and K. Samwer, Phys. Rev. Lett. 71, 2331 (1993); S. Jin, T. H. Tiefel, M. McCormack, R. A. Fastnacht, R. Ramesh, and L. H. Chen, Science 264, 413 (1994).

[2] For a review, see Colossal Magnetoresistance Oxides, edited by Y. Tokura (Gordon and Breach, London, 1999).

[3] A. Urushibara, Y. Moritomo, T. Arima, A. Asamitsu, G. Kido, and Y. Tokura, Phys. Rev. B 51, 14103 (1995).

[4] P. Dai, H. Y. Hwang, J. Zhang, J. A. Fernandez-Baca, SW. Cheong, C. Kloc, Y. Tomioka, and Y. Tokura, Phys. Rev. B 61, 9553 (2000).

[5] P. G. Radaelli, G. Iannone, M. Marezio, H. Y. Hwang, SW. Cheong, J. D. Jorgensen, and D. N. Argyriou, Phys.
Rev. B 56, 8265 (1997).

[6] V. Laukhin, J. Fontcuberta, J. L. Garcia-Muñoz, and X. Obradors, Phys. Rev. B 56, R10009 (1997).

[7] A. J. Millis, P. B. Littlewood, and B. I. Shraiman, Phys. Rev. Lett. 74, 5144 (1995); A. J. Millis, B. I. Shraiman, and R. Mueller, ibid. 77, 175 (1996).

[8] S. J. L. Billinge, R. G. DiFrancesco, G. H. Kwei, J. J. Neumeier, and J. D. Thompson, Phys. Rev. Lett. 77, 715 (1996); K. H. Kim, J. Y. Gu, H. S. Choi, G. W. Park, and T. W. Noh, ibid. 77, 1877 (1996); M. Jaime, H. T. Hardner, M. B. Salamon, M. Rubinstein, P. Dorsey, and D. Emin, ibid. 78, 951 (1997).

[9] C. H. Booth, F. Bridges, G. H. Kwei, J. M. Lawrence, A. L. Cornelius, and J. J. Neumeier, Phys. Rev. Lett. 80, 853 (1998).

[10] S. Shimomura, N. Wakabayashi, H. Kuwahara, and Y. Tokura, Phys. Rev. Lett. 83, 4389 (1999); L. VasiliuDoloc, S. Rosenkranz, R. Osborn, S. K. Sinha, J. W. Lynn, J. Mesot, O. H. Seeck, G. Preosti, A. J. Fedro, and J. F. Mitchell, ibid. 83, 4393 (1999).

[11] D. Louca, T. Egami, E. L. Brosha, H. Röder, and A. R. Bishop, Phys. Rev. B 56, R8475 (1997).

[12] P. Dai, J. A. Fernandez-Baca, N. Wakabayashi, E. W. Plummer, Y. Tomioka, and Y. Tokura, Phys. Rev. Lett. 85, 2553 (2000); C. P. Adams, J. W. Lynn, Y. M. Mukovskii, A. A. Arsenov, and D. A. Shulyatev, ibid. 85, 3954 (2000); C. S. Nelson, M. v. Zimmermann, Y. J. Kim, J. P. Hill, Doon Gibbs, V. Kiryukhin, T. Y. Koo, S-W. Cheong, D. Casa, B. Keimer, Y. Tomioka, Y. Tokura, T. Gog, and C. T. Venkataraman, Phys. Rev. B 64, 174405 (2001).

[13] T. Y. Koo, V. Kiryukhin, P. A. Sharma, J. P. Hill, and S-W. Cheong, Phys. Rev. B 64, 220405(R) (2001).

[14] Nanoscale structural correlations have also been found recently in bi-layered manganites, and they were associated with charge-density-wave fluctuations. However, the wave vector of these correlations is very different (rotated $45^{\circ}$ ) from the wave vector of the correlations in the perovskite manganites discussed here, and therefore it is unclear whether these two phenomena are related. See B. J. Campbell, R. Osborn, D. N. Argyriou, L. Vasiliu-Doloc, J. F. Mitchell, S. K. Sinha, U. Ruett, C. D. Ling, Z. Islam, and J. W. Lynn, Phys. Rev. B 65, 014427 (2001).

[15] V. Kiryukhin, T. Y. Koo, A. Borissov, Y. J. Kim, C. S. Nelson, J. P. Hill, D. Gibbs, and S-W. Cheong, Phys. Rev. B 65, 094421 (2002).

[16] J. M. Zuo, and J. Tao, Phys. Rev. B 63, 060407(R), (2001).

[17] Note, an alternative model describing the lowtemperature state of half-doped manganites as ordering of Zener polarons has been proposed recently, see A. Daoud-Aladine, J. Rodriguez-Carvahal, L. PinsardGaudart, M. T. Fernández-Diaz, and A. Revcolevschi, Phys. Rev. Lett. 89, 097205 (2002). We, however, keep referring to this state as CE-type charge and orbital ordered, because this assignment is dominant in the current literature.

[18] For a review, see E. Dagotto, T. Hotta, and A. Moreo, Phys. Rep. 344, 1 (2001).

[19] L. M. Rodriguez-Martinez, and J. P. Attfield, Phys. Rev. B 54, 15622 (1996). 
[20] D. I. Khomskii, and K. I. Kugel, Europhys. Lett. 55, 208 (2001).

[21] H. Kuwahara, T. Okuda, Y. Tomioka, A. Asamitsu, and Y. Tokura, in Science and Technology of Magnetic Oxides, edited by M. Hundley, J. Nickel, R. Ramesh, and Y. Tokura, MRS Symp. Proc. No. 494 (Materials Research Society, Pittsburgh, 1998), p. 83.

[22] P. G. Radaelli, D. E. Cox, M. Marezio, and S-W. Cheong, Phys. Rev. B 55, 3015 (1997).

[23] D. N. Argyriou, J. W. Lynn, R. Osborn, B. Campbell, J. F. Mitchell, U. Ruett, H. N. Bordallo, A. Wildes, and C. D. Ling, Phys. Rev. Lett. 89, 036401 (2002).

[24] M. Jaime, P. Lin, S. H. Chun, and M. B. Salamon, Phys. Rev. B 60, 1028 (1999).

[25] S. Shimomura, T. Tonegawa, K. Tajima, N. Wakabayashi, N. Ikeda, T. Shobu, Y. Noda, Y. Tomioka, and Y. Tokura, Phys. Rev. B 62, 3875 (2000); R. Kajimoto, H. Yoshizawa, Y. Tomioka, and Y. Tokura, ibid. 63, 212407 (2001).

[26] R. Kajimoto, H. Yoshizawa, H. Kawano, H. Kuwahara, Y. Tokura, K. Ohoyama, and M. Ohashi, Phys. Rev. B 60, 9506 (1999).

[27] Q. Huang, A. Santoro, J. W. Lynn, R. W. Erwin, J. A. Borchers, J. L. Peng, K. Ghosh, and R. L. Greene, Phys. Rev. B 58, 2684 (1998).

[28] J. F. Mitchell, D. N. Argyriou, C. D. Potter, D. G. Hinks, J. D. Jorgensen, and S. D. Bader, Phys. Rev. B 54, 6172 (1996).

[29] Y. Tomioka, A. Asamitsu, and Y. Tokura, Phys. Rev. B 63, 024421 (2000).

[30] J. Mira, J. Rivas, L. E. Hueso, F. Rivadulla, M. A. López Quintela, N. A. Señaris Rodriguez, C. A. Ramos, Phys. Rev. B 65, 024418 (2001). 